\title{
Desarrollo profesional de los docentes de educación secundaria: estudio sobre el nivel de mejora de las habilidades sociales
}

\author{
Eva María Torrecilla-Sánchez, Susana Olmos \\ Migueláñez y María José Rodríguez-Conde
}

\begin{abstract}
Resumen
La formación a lo largo de la vida es un criterio clave en el desarrollo profesional de los docentes de educación secundaria, colectivo que debe adaptarse a las peculiaridad del contexto y momento temporal en que se integran. En el siglo XXI el domino de las habilidades sociales para la gestión de las interacciones en su centro de trabajo es un componente principal en sus necesidades. Conscientes de esta realidad social se diseña e implementa un proceso formativo cooperativo orientado al colectivo de docentes de educación secundaria y centrado en la resolución de conflictos, de cara a mejorar sus habilidades sociales. Constatar la eficacia del proceso formativo requiere un diseño cuasiexperimental pre-postest, utilizando como instrumento de medida la Escala de Habilidades Sociales de Gismero (2000). En el proceso formativo se contó con una muestra 34 docentes en activo de la provincia de Salamanca (España). Los resultados obtenidos, establecen diferencias significativas a favor de la aplicación del programa en dos de los seis factores de medida, junto a la variable global. A su vez, la variable género supone una característica diferenciadora para el nivel de habilidades sociales de los sujetos. Se concluye con la necesidad de implementar programas formativos específicos para mejorar las competencias de los docentes desde su desarrollo profesional.
\end{abstract}

Palabras clave:

evaluación de programas; formación docente; habilidades sociales; resolución de conflictos. 


\title{
Desenvolvimento profissional dos professores do ensino secundário: estudo sobre habilidades sociais
}

\begin{abstract}
Resumo: A formação ao longo da vida é um critério fundamental no desenvolvimento profissional dos professores do ensino secundário, colectivo que devem adaptar-se à particularidade do contexto e tempo em que estão integrados. No século XXI, o domínio de habilidades sociais para o gestão de interações entre os diversos atores da comunidade escolar é um importante elemento de suporte para a coexistência. Consciente desta realidade social, este estudo foi concebido e implementado num grupo cooperativo no processo de aprendizagem de professores do ensino secundário e focado na resolução de conflitos, com o intuito de melhorar suas habilidades sociais. Para verificar a eficácia do processo que a formação, tem havido uma avaliação quasi-experimental de design pré-pós-teste, e usado como um instrumento de avaliação a Escala de Habilidades Sociais de Gismero (2000). No processo de formação, que envolveu uma amostra de 34 professores ativos na província de Salamanca (Espanha). Os resultados mostram diferenças significativas a um nível de significância de 0,05, para o programa, em duas das seis dimensões. Por sua vez, a variável género aparece como característica diferenciadora ao nível no que concerne às habilidades sociais. Conclui-se, que existe a necessidade de implementar programas específicos de formação para melhorar as competências dos professores a partir de uma abordagem de desenvolvimento profissional.
\end{abstract}

Palavras-chave: avaliação de programas; formação de professores; habilidades sociais; resolução de conflitos.

\section{Secondary education teachers' professional development: a study on social skills}

Abstract: Lifelong learning is a key element in the professional development of secondary education teachers, a collective that must adapt to the characteristics of the context and time in which they are integrated. In the $21^{\text {st }}$ century, the command of social skills for the management of the interactions among the different community sectors within the school is a very important issue to support coexistence. Bearing this reality in mind, this study designs and implements a cooperative training process aimed at secondary education teachers and focused on conflict resolution, with the objective of enhancing their social skills. The efficacy of the training process was verified through the design of a quasi-experimental pretest-posttest assessment, using the Gismero's Social Skills Scale (2000). There were 34 teachers from the province of Salamanca (Spain) involved in the training process. The results obtained show significant differences (s.l. .05) in favour of the programme in two out of the six dimensions. In its turn, the variable gender appears as a differentiating feature regarding the individuals' social skill level. We conclude with the need to implement specific training programmes for the improvement of teacher social skills from a professional development approach.

Key words: programme assessment; teacher training; social skills; conflict resolution.

\section{Développement professionnel des enseignants de l'enseignement secondaire: une étude des compétences sociales}

Resumé: La formation tout au long de la vie est un critère clé dans le développement professionnel des enseignants de l'éducation secondaire, un collective qui doit s'adapter à la particularité du contexte et le temps où il est intégré. Au XXI e siècle, le domaine des compétences sociales pour gérer les interactions entre les secteurs de la communauté dans un centre educatif est un élément important de soutien à la coexistence. Conscients de cette réalité sociale, dans cette étude on conçoit et on met en oeuvre un processus d'apprentissage coopératif orienté vers le collectif des enseignants de l'enseignement secondaire et axé sur la résolution des conflits, afin d'améliorer leurs compétences sociales. Pour vérifier l'efficacité du processus de formation il a été nécessaire une évaluation quasi-expérimentale de conception pré-posttest, et on a utilisé comme un instrument de mesure l'Echelle de Competences sociales Gismero (2000). Dans le processus de formation, il y avait un échantillon de 34 enseignants actifs dans la province de Salamanque (Espagne). Les résultats montrent des différences significatives au niveau de .05 de signification, pour le programme, dans deux des six dimensions. À son tour, la variable de genre apparaît comme la différenciation caractéristique pour le niveau de compétences sociales des sujets. On conclut à la nécessité de mettre en œuvre des programmes de formation spécifiques visant à améliorer les compétences des enseignants à partir d'une approche de développement professionnel.

Mots-clés: évaluation du programme; formation des enseignants; compétences sociales; résolution des conflits. 


\section{Introducción}

Los docentes de educación secundaria son uno de los colectivos en los que mayor relevancia tiene la formación a lo largo de la vida. Su importancia radica en la propia identidad de su profesión; en este sentido, la identidad profesional comprende el resultado, tanto en la percepción de la propia profesión, como el desempeño diario en la misma (Bolívar, 2007).

La realidad de este colectivo, concluye que, en la identidad profesional docente el desarrollo profesional es clave, al comprender un proceso de integración en la práctica propia o de otros profesionales con el fin de analizar y teorizar sobre el trabajo docente y vincularlo con aspectos sociales, cultures y políticos.

\section{Por qué el desarrollo profesional de los docentes de educación secundaria}

Como proceso constructivo la identidad profesional incluye cambios constantes, influenciados tanto por aspectos personales, como sociales. Traduciéndose en un proceso de socialización profesional que interviene por un lado, en la adquisición de la cultura profesional y, por otro, en las normas, reglas, habilidades, conocimientos y valores asociados a la función docente (Bolívar, 2007). Este proceso comienza con los primeros contactos con el sistema educativo, desde las etapas de estudiante (González Sanmamed, 2009; Marcelo \& Vaillant, 2009), hasta su desarrollo profesional en los centros como docentes. Al tratarse de un proceso de socialización, intervienen elementos interrelacionados que marcan la carrera docente, como son las historias de vida antes, durante y después de la formación inicial, las diversas fases de la carrera formativa en las Universidades, las relaciones que se establecen con otros docentes en los centros educativos y, por supuesto, la autoreflexión sobre la práctica y los intercambios comunicativos con especialistas (Fernández Cruz, 2006; Vidiella Pagés, 2011). Es por ello, que siguiendo a Grossman (1992) se diferencian dos fases: (a) Inicial de Formación y (b) Desarrollo Profesional.

El porqué del desarrollo profesional docente radica en ambas etapas, al comprender esta formación diferentes aspectos relacionados con, la historia docente, las características sociales y el contexto en el que se desarrolla (Marcelo \& Vaillant, 2009). En este sentido, es relevante analizar los niveles formativos por la carga que cada elemento clave tiene en los mismos.

\section{Características relevantes de la formación inicial de los docentes}

En la formación inicial se adquieren conocimientos, competencias y habilidades para la primera toma de contacto en las aulas dentro de un contexto universitario y con un rol de estudiante. En esta etapa se produce la primera "relación" con el centro educativo como docente pero desde un sentido formativo. En este periodo debe 
adquirir diferentes estrategias, competencias y conocimientos, desde el propio marco de su actividad diaria. Esta fase de contacto inicial es fundamental en los docentes de educación secundaria que tienen entre sus principales cometidos, transmitir una serie de contenidos -conocimiento teórico-; sin embargo, para ser docente no basta con conocer una disciplina concreta. Es necesario, también, el conocimiento educativo -conocimiento aplicado a la práctica, formación pedagógica- (González Gallego, 2010). El dilema se plantea en el valor que se otorga a cada uno de los saberes que debe dominar un docente: (1) el conocimiento teórico y (2) aplicado a la práctica. La forma que se da al proceso formativo en relación con ambos saberes ha derivado en dos perspectivas diferentes: modelo simultáneo y modelo sucesivo.

El modelo sucesivo sustenta una formación dividida, en la que el mayor "peso" lo configuran los estudios basados en una disciplina por encima del conocimiento aplicado a la práctica (Barberá, 2010; Bolívar, 2007; Esteve, 2006). Comprende dos fases formativas divididas en tiempo y forma, en las que se otorga mayor relevancia a la formación en conocimiento teórico, que a la propia función como futuro docente. La ruptura entre ambas y la descontextualización se traduce en una identidad profesional no acorde a la profesión docente.

En cuanto al modelo simultáneo promueve la unión del conocimiento en la disciplina y metodología docente en un mismo tiempo (Barberá, 2010; Bolívar, 2007; Esteve, 2006); su puesta en práctica implica la formación de los estudiantes en una disciplina, a la vez que adquieren conocimientos aplicados a la práctica sobre la función docente. Integra la formación pedagógica en las diversas titulaciones (Matemáticas, Bilogía, Historia, etc.). En este caso, la identidad se va formando acorde a la función docente, a la vez que, no supone un elemento de segundo orden si no de primero; es decir, el estudiante que quiere ser docente se lo plantea durante su formación, no ante la falta de otras salidas profesionales.

En Europa, el más común es el modelo simultáneo, aunque existen países que lo utilizan de forma combinada con el sucesivo, como puede observarse en la Tabla 1.

Tabla 1

Modelos formativos para docentes de Educación Secundaria en Europa

\begin{tabular}{|c|c|c|}
\hline $\begin{array}{l}\text { Modelo } \\
\text { formativo }\end{array}$ & Países Europeos & \\
\hline $\begin{array}{l}\text { Modelo } \\
\text { Simultáneo }\end{array}$ & $\begin{array}{l}\text { Alemania, Bélgica, Dinamarca, Países Bajos, } \\
\text { Portugal, Polonia, República Checa, Estonia, } \\
\text { Letonia, Hungría, Rumania, Eslovaquia. }\end{array}$ & \multirow{2}{*}{$\begin{array}{c}\text { Irlanda, Australia, Finlandia, } \\
\text { Suecia, Eslovenia, Reino } \\
\text { Unido, Islandia, Noruega, } \\
\text { Lituania, Malta. }\end{array}$} \\
\hline $\begin{array}{l}\text { Modelo } \\
\text { Sucesivo }\end{array}$ & $\begin{array}{l}\text { España, Francia, Italia, Grecia, Luxemburgo, } \\
\text { Lituania, Bulgaria, Chipre. }\end{array}$ & \\
\hline
\end{tabular}

(Elaborado a partir de Eurydice, 2003) 
En conclusión, el modelo bajo el que se forme el docente establece una identidad de partida arraigada a la disciplina académica o la interacción entre la función docente y la disciplina académica.

\section{Desarrollo profesional docente}

El desarrollo profesional, supone el perfeccionamiento dentro del propio centro educativo, donde cambia tanto el contexto de interacción, como el rol a desempeñar. En esta fase se integra la formación en ejercicio, también conocida como: formación continua, formación permanente, formación en servicio y actualización (González Sanmamed, 2009; Imbernón, 2014; Bolívar, 2007; Fernández Cruz, 2006).

Al tratarse el desarrollo profesional de un proceso vivenciado desde la propia profesión docente, hace referencia a un concepto amplio en el que se integran tanto procesos diferenciados de formación continua, como todas aquellas experiencias y factores vinculados con el salario, la demanda laboral, el clima laboral y social de los centros, la promoción dentro de la profesión, las estructuras jerárquicas, la carrera docente, el periodo vacacional, relaciones con las familias, etc. (Imbernón, 2014). Experiencias que hacen "crecer" al profesorado, configurando una función completa que le permite transferir sus aprendizajes, valores, creencias, etc., al resto de colectivo educativo relacionado con él. La formación continua, configura uno de los momentos puntuales en los que el docente decide formar parte de un procedimiento consciente de aprendizaje, que potencia e influye en su desarrollo profesional.

A su vez, este proceso debe integrar la capacidad de indagación sobre el contexto de trabajo -la clase- con intención de identificar necesidades, buscar soluciones, e incorporarlas al aula. Comprende una transformación paulatina que comienza a lo largo de la formación inicial -con la indagación al margen de la formación reglada- continuando durante la vida ocupacional (Fernández Cruz, 2006). Es decir, ante las carencias que se observen en y para el desempeño de la profesión, ser capaz de buscar las respuestas de manera autónoma ya sea antes de la entrada en el contexto educativo como docente o bien dentro del mismo.

La unión e importancia de la formación continua en el desarrollo profesional radica en la necesidad de reflexión sobre la práctica y el trabajo colaborativo, tanto para el intercambio de experiencias, como para provocar procesos de comunicación (Imbernón, 2014).

La organización de esta etapa formativa se ha visto configurada por modelos heterogéneos de desarrollo, promovidos, en muchas ocasiones, por las necesidades sociales del momento o corriente pedagógica predominante. Motivando, a su vez, contenidos formativos acordes a las necesidades cambiantes de la población en formación y llegando a la constitución de centros específicos para el desarrollo profesional docente, con la intención de poder atender como se merece a este colectivo. 
El desarrollo profesional docente se organiza con criterios dispares, según el fin que persiga. Esta situación refleja la delimitación de clasificaciones diferentes dependiendo del autor que se considere. Estas propuestas establecen la elaboración de una clasificación, no excluyente, de modelos de desarrollo profesional atendiendo a (Fernández Cruz, 2006; Imbernón, 2014; Martínez Bonafé, 2010):

1. Actividad formativa: la "forma" de la actividad

a. Seminario de elaboración de

i. Materiales

ii. Proyectos

b. Cursos

i. De divulgación

ii. Prácticos

iii. De formación

c. Jornadas y encuentros científico-experienciales

d. Conferencias

e. Intercambios y experiencias formativas

2. Proceso: el trabajo del docente para formarse

f. Reflexión

g. Investigación o indagación

h. Observación-evaluación

3. Interacción de los implicados: organización interdependiente o independiente por parte de los participantes para llegar al fin formativo

i. Formación autónoma

j. Formación cooperativa o colaborativa

k. Comunidad formativa

I. Comunidad de aprendizaje

4. Contenidos: el punto principal de trabajo

m. Las adquisiciones como elemento formativo principal

n. El proceso como itinerario de formación

o. El análisis de experiencias para la formación

5. Contexto formativo: necesidad del propio centro o una propuesta externa

p. Modelos centrados en la escuela

q. Modelos externos a la escuela

Por su parte, las temáticas que se integran son independientes del modelo que se implementa. No obstante, deben atender a las necesidades reales de los docentes. En la actualidad, las características del contexto promueve temas centrados en: el desarrollo emocional, la comunicación efectiva, convivencia en las instituciones, fracaso escolar, metodologías diferenciadas de acuerdo con el grupo, actuaciones prácticas para 
el desarrollo de las disciplinas, detección de necesidades y atención a necesidades diversas (Imbernón, 2014). Estos contenidos versan sobre la población de nuestros días, según las exigencias del entorno. Se dirigen al cambio de actitudes de los docentes y permiten configurar intereses y necesidades comunes, tanto para receptores, como motivadores del proceso de enseñanza-aprendizaje.

En definitiva, el sentido de la formación docente radica, tanto en la formación inicial como, en el desarrollo profesional, ambos periodos suponen aprendizajes importantes para la constitución de su identidad al permitirle adaptarse a las características sociales y del contexto de trabajo. Por ello, la formación continua constituye un elemento relevante para los docentes si se orienta a la adquisición de competencias y estrategias. De ahí, la necesidad de implementar procesos formativos validados que contribuyan al perfeccionamiento docente.

\section{Método}

El presente estudio de corte cuasiexperimental pretest-postest, pretende comprobar el impacto de una actividad formativa, en formato de curso colaborativo, con metodología blearning, en resolución de conflictos acerca del nivel autopercibido de habilidades sociales de un grupo de docentes de educación secundaria. Para ello, se tienen en cuenta las variables independientes que pueden influir sobre el nivel de desarrollo profesional alcanzado en el proceso formativo, destacando la titularidad del centro, el sexo y los años de experiencia como docentes.

En definitiva, se pretende conocer la adecuación o no de implementar una acción formativa en la fase de desarrollo profesional docente, en la que la variable de estudio son las habilidades sociales.

\section{Muestra}

El programa de resolución de conflictos se implementa en el curso 2013-14 destinado a docentes de educación secundaria de la provincia de Salamanca (España). Con la finalidad de seleccionar la muestra se contacta con centros del entorno y se les oferta el programa, teniendo en cuenta la titularidad del centro (público y concertado). Esta variable categórica se considera relevante al suponer una variable del contexto que puede explicar cambios en los resultados, tanto en la eficacia de un proceso, como en el nivel de rendimiento de los estudiantes (Choi y Calero, 2012; De la Orden y Jornet, 2012).

El acceso a la muestra se lleva a cabo mediante el equipo directivo de varios centros. Tras la respuesta positiva de dos centros (uno de titularidad pública y otro de privada-concertada), se deja libertad, para que sean ellos quienes determinen la selección de docentes.

El muestro no probabilismo, incidental, por criterios implementado en el proceso concluye con una muestra representativa en cuanto a la titularidad del centro $(52.9 \%$ 
centro público y $47.1 \%$ centro privado-concertado) y a la variable sexo (50\% de hombres y $50 \%$ de mujeres). En lo que respecta a los años de docencia el menor porcentaje de la muestra se corresponde con docentes noveles (1 a 5 años con un 5.9\%), mientras que en el resto de dimensiones los porcentajes fluctúan siempre alrededor del $30 \%$. En definitiva, la distribución muestral, determinada por 34 docentes, representa a la realidad educativa estudiada (tabla 2).

Tabla 2.

Distribución de la muestra

\begin{tabular}{ccccccccc} 
& \multicolumn{2}{c}{ Sexo } & \multicolumn{2}{c}{ Titularidad del centro } & \multicolumn{3}{c}{ Años de docencia } \\
\cline { 2 - 9 } & Hombre & Mujer & Público & Concertado & $1-5$ & $6-15$ & $15-25$ & Más de 25 \\
\hline $\mathrm{f}$ & 17 & 17 & 18 & 16 & 2 & 11 & 10 & 11 \\
\hline$\%$ & 50.0 & 50.0 & 52.9 & 47.1 & 5.9 & 32.4 & 29.4 & 32.4 \\
\hline
\end{tabular}

Fuente: Elaboración propria

\section{Instrumento}

El instrumento de medida para el nivel de Habilidades Sociales autopercibido es la "Escala de Habilidades Sociales" (EHS) de Gismero (2000). Esta herramienta explora la conducta de las personas en situaciones cotidianas constatando la influencia de las habilidades sociales en las mismas. La información se recoge mediante 33 ítems -28 redactados en sentido inverso y 5 en sentido positivo-, que hacen referencia a seis factores: F1. Autoexpresión en situaciones sociales; F2. Defensa de los propios derechos como consumidor; F3. Expresión de enfado o disconformidad; F4. Decir no y cortar interacciones; F5. Hacer peticiones; F6. Iniciar interacciones positivas con el sexo opuesto. Las características definitorias del instrumento de medida, ateniendo a cada uno de los factores anteriores, comprende 6 variables de estudio.

La escala permite diferenciar entre sujetos con alta y baja habilidad social, atendiendo a baremos diferenciados para adultos y jóvenes, a la vez que hombres y mujeres. Baremos que permiten transformar las puntuaciones directas en percentiles facilitando la interpretación de los datos.

La fiabilidad del instrumento, calculada por la autora mediante el procedimiento de covarianza entre todos los ítems, obtiene un valor de alpha de Cronbach de .88; es decir, ofrece indicios de estabilidad alta entre las puntuaciones de la escala. Dicha estabilidad es corroborada con la muestra de estudio, en la medida pretest, con un valor de .89.

\section{Procedimiento}

La hipótesis sobre la que versa el presente estudio se concreta en "un modelo de evaluación y desarrollo de competencias en resolución de conflictos dentro del 
programa de formación permanente del profesorado de educación secundaria, basado en el uso de las TIC y en el trabajo cooperativo entre profesores, contribuirá a mejorar su nivel de competencias en habilidades sociales". Para lo que se formula la siguiente hipótesis de trabajo "no existen diferencias significativas en la dimensión habilidades sociales, ni en los factores que la configuran, entre la medida pretest-postest para los docentes participantes en la acción formativa en resolución de conflictos". Tras el estudio de la normalidad, mediante la prueba de Kolmogorov-Smirnov, utilizando la diferencia entre los factores del postest y prestest, se corrobora el ajuste a la normalidad de la muestra (tabla 3). Por lo que se opta, para dar respuesta a la hipótesis, por la prueba t de Student (n.s .05) para muestras relacionadas, junto al tamaño del efecto, mediante el cálculo de la d de Cohen (Cohen, 1969).

Con el fin de profundizar en los análisis, teniendo en cuenta las características de la muestra, se generan tres sub-hipótesis relacionadas con dos variables independientes: sexo y titularidad del centro. La primera sub-hipótesis hace referencia a las diferencias atendiendo al sexo, la segunda a la titularidad y la tercera a la interacción entre ambas. En este caso, se plantea un problema estadístico al reducirse el tamaño de la muestra, para cada variable -ver tabla 2-. Por otra parte, la existencia de variables cualitativas junto a una cuantitativa, a la vez que varias variables de estudio (tres), conlleva el análisis de varianza con un factor (ANOVA), previo a la misma se estudia la normalidad mediante la prueba de Levene, con el fin de constatar si el tamaño muestral permite dicho análisis. Constatado el ajuste de normalidad, se establece la idoneidad para el análisis de varianza.

Los análisis estadísticos se llevaron a cabo mediante el programa SPSS v. 23 (licencia de la Universidad de Salamanca).

Tabla 3.

Estudio de la normalidad mediante la prueba Kolmogorov-Smirnov

\begin{tabular}{cccc}
\hline D Factor & \multicolumn{3}{c}{ Kolmogorov-Smirnov } \\
\cline { 2 - 4 } (Postest-Prestest) & $\mathrm{d}$ & $\mathrm{gl}$ & $\mathrm{p}$ \\
\hline D_F1 & .129 & 34 & .163 \\
\hline D_F2 & .089 & 34 & $>.200$ \\
\hline D_F3 & .084 & 34 & $>.200$ \\
\hline D_F4 & .096 & 34 & $>.200$ \\
\hline D_F5 & .099 & 34 & $>.200$ \\
\hline D_F6 & .078 & 34 & $>.200$ \\
\hline D_Percentil Global & .106 & 34 &
\end{tabular}

Fuente: Elaboración propria 


\section{Resultados}

Los resultados obtenidos, que permiten dar respuesta a la hipótesis de estudio, parten de un análisis descriptivo de la variable habilidades sociales. En este sentido, se lleva a cabo el estudio de la media y la desviación típica con el fin de constatar, a priori, si existen diferencias tras la aplicación del programa. Este primer análisis exploratorio muestra diferencias a favor del programa, al constatarse medias mayores en el postest que en el prestest. Es reseñable, en este sentido, el F5. Hacer peticiones. En el gráfico 1 se comprueba la mayor diferencia en referencia a todos los factores de estudio. Por otra parte, en lo que respecta a la dispersión de los datos (desviación típica) se constata cierta homogeneidad en los datos (tabla 4).

\section{Gráfico 1.}

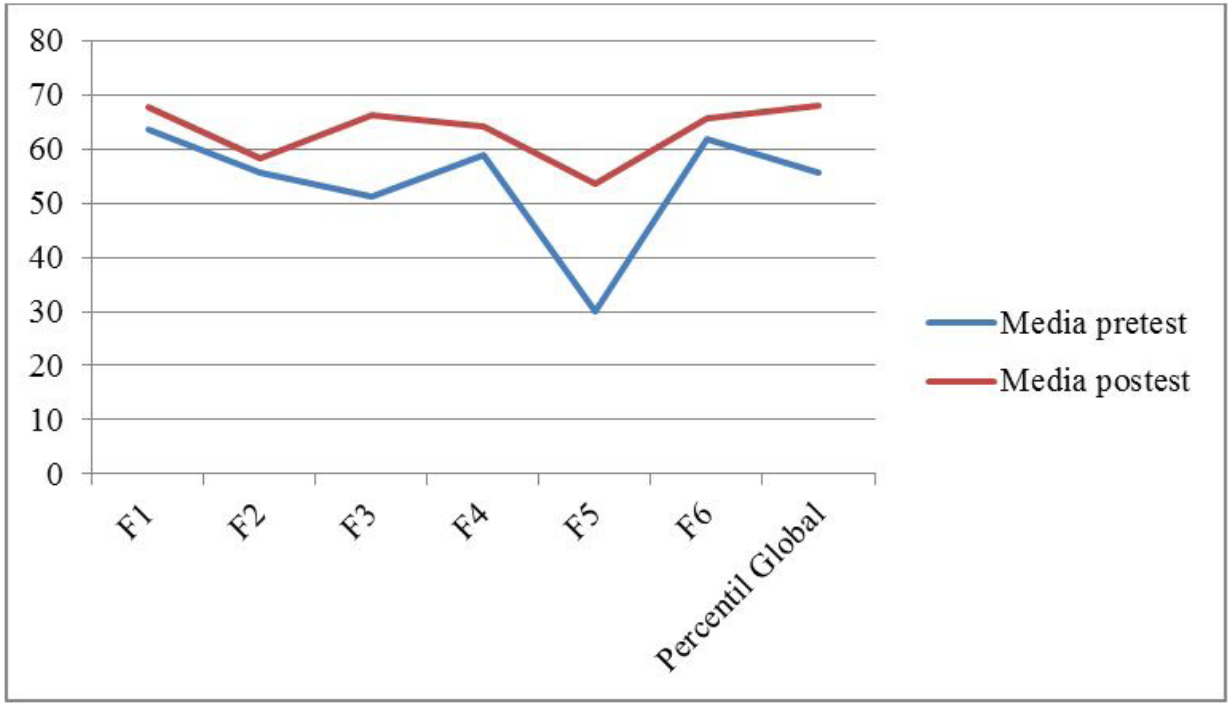

Media de las puntuaciones en el prestest-postest

El contraste de hipótesis con la prueba t de Student junto al tamaño del efecto con la d de Cohen (tabla 4), permite corroborar que tras la aplicación del programa los participantes mejoran sus habilidades sociales. Esta afirmación se apoya en los resultados obtenidos en el percentil global $(p=.007)$ con un tamaño del efecto entre pequeño y moderado $(\mathrm{d}=.457)$. No obstante, el resultado global se constata por el peso que dos de los factores ejercen sobre el total. Se trata del F3. Expresión de enfado o disconformidad y el F5. Hacer peticiones. 
El estudio pormenorizado en ambos factores mediante la diferencia de medias (n.s .05) y la influencia del tamaño del efecto en el F3 es significativa ( $p$.003) apoyada en un tamaño del efecto moderado ( $d=$.609). En el F5, la significatividad es mayor (p. 000) con un tamaño del efecto alto $(\mathrm{d}=.859)$.

El cambio en estos factores se relaciona directamente con las competencias trabajadas en el curso. El F3 recoge información sobre la gestión de situaciones conflictivas y la regulación emocional, por su parte, el F5 integra la asertividad en la relación con los otros y afrontar o evitar conflictos. Estas competencias se trabajan de forma activa en el curso, lo que implica que, tras los resultados obtenidos, el curso tiene influencia positiva sobre los participantes.

Tabla 4.

Análisis descriptivo y contraste de hipótesis de los factores de la variable medida

\begin{tabular}{|c|c|c|c|c|c|c|c|}
\hline \multirow[b]{2}{*}{ Factores } & \multicolumn{2}{|c|}{ Prestest } & \multicolumn{2}{|c|}{ Postest } & \multirow[b]{2}{*}{$t$} & \multirow[b]{2}{*}{$p$} & \multirow[b]{2}{*}{$d$} \\
\hline & Media & $\begin{array}{c}\text { Desviación } \\
\text { típica }\end{array}$ & Media & $\begin{array}{c}\text { Desviación } \\
\text { típica }\end{array}$ & & & \\
\hline $\mathrm{F} 1$ & 63.56 & 23.83 & 67.71 & 27.23 & -1.13 & .268 & .152 \\
\hline $\mathrm{F} 2$ & 55.71 & 30.60 & 58.24 & 28.30 & -0.46 & .650 & .089 \\
\hline F3 & 51.12 & 30.53 & 66.26 & 24.85 & -3.20 & .003 & .609 \\
\hline $\mathrm{F} 4$ & 58.91 & 31.18 & 64.09 & 28.60 & -0.91 & .371 & .181 \\
\hline F5 & 29.88 & 25.92 & 53.68 & 27.72 & -6.71 & .000 & .859 \\
\hline F6 & 61.85 & 27.90 & 65.71 & 29.70 & -0.83 & .413 & .130 \\
\hline $\begin{array}{c}\text { Percentil } \\
\text { Global }\end{array}$ & 55.65 & 29.89 & 68.18 & 27.41 & -2.86 & .007 & .457 \\
\hline
\end{tabular}

Fuente: Elaboración propria

Determinada la evidencia de cambios en la variable habilidades sociales, se estudia si las variables categóricas tienen influencia en los resultados globales. En este sentido, los resultados del análisis de varianza de medidas repetidas se agrupan en relación a las variable sexo, titularidad del centro y la interacción entre ambas. En la tabla 5 se constata cómo no existen diferencias estadísticamente significativas (n.s .05) ateniendo a la titularidad del centro o a la interacción entre titularidad y sexo. En lo que respecta a la variable sexo en el factor 4 y 6 parecen existir diferencias. Ante estos datos se estudia el tamaño del efecto mediante $\mathrm{h}^{2}$. Atendiendo a los valores (Becker, 2000) que toma cada uno de los factores en este estadístico de medida se comprueba como en tres factores el efecto es moderado (F1, F4 y F6). Corroborando ambos estadísticos parece que existen diferencias en habilidades sociales en la variable sexo, diferencias claras en los factores F4 y F6, y más limitadas en el F1. 
Tabla 5.

Análisis de varianza de dos factores (sexo y titularidad), con medidas repetidas (pre-postest) para cada factor de medida

\begin{tabular}{|c|c|c|c|c|c|c|c|c|c|c|c|}
\hline \multirow{2}{*}{$\begin{array}{l}\text { Factores } \\
\text { (Prestest y } \\
\text { Postest) }\end{array}$} & \multicolumn{2}{|c|}{$\begin{array}{c}\text { Prueba de } \\
\text { homogeneidad } \\
\text { (Levene) }\end{array}$} & \multicolumn{3}{|c|}{ Sexo } & \multicolumn{3}{|c|}{ Titularidad centro } & \multicolumn{3}{|c|}{$\begin{array}{l}\text { Titularidad del } \\
\text { centro*Sexo }\end{array}$} \\
\hline & $\begin{array}{l}\text { F (Pre) } \\
\text { (Post) }\end{array}$ & $\begin{array}{l}\text { p (Pre) } \\
\text { (Pos) }\end{array}$ & F & $\mathrm{p}$ & h2 & $\mathrm{F}$ & $\mathrm{p}$ & h2 & $\mathrm{F}$ & $\mathrm{p}$ & h2 \\
\hline $\mathrm{F} 1$ & 0.16 & .925 & 4.04 & .054 & .111 & 0.75 & .394 & .021 & 0.02 & .903 & .000 \\
\hline $\mathrm{F} 2$ & 1.70 & .188 & 0.12 & .736 & .004 & 0.10 & .759 & .003 & 0.00 & .998 & .007 \\
\hline F3 & 1.92 & .148 & 2.28 & .141 & .054 & 0.07 & .787 & .002 & 0.29 & .593 & .010 \\
\hline $\mathrm{F} 4$ & 0.45 & .719 & 4.85 & .035 & .140 & 0.05 & .834 & .001 & 0.16 & .691 & .005 \\
\hline F5 & 0.93 & .438 & 1.50 & .231 & .019 & 2.56 & .120 & .032 & 0.07 & .801 & .000 \\
\hline F6 & 1.92 & .148 & 4.22 & .049 & .119 & 0.09 & .761 & .003 & 0.45 & .508 & .013 \\
\hline $\begin{array}{l}\text { Percentil } \\
\text { Global }\end{array}$ & 0.27 & .850 & 2.69 & .112 & .066 & 0.13 & .722 & .003 & 0.02 & .899 & .000 \\
\hline
\end{tabular}

Fuente: Elaboración propria

Los resultados obtenidos en el análisis de varianza para la variable sexo encaminan un estudio más pormenorizado de los factores en esta variable. En este sentido, se lleva a cabo un análisis descriptivo para comprobar cómo se comporta dicha variable.

La tabla 6 permite constatar como las mujeres que participaron en el curso, tanto en el prestest como en el postest, suelen obtener puntuaciones algo inferiores a los hombres. Si bien es cierto, las diferencias mayores ( $D>10)$, ya sea en el prestest o en el postest, incluso en ambos, se producen en los tres factores resaltados tras el análisis de varianza. Se recuerda que dichos factores hacen referencia a: F1. Autoexpresión en situaciones sociales; F4. Decir no y cortar interacciones; F6. Iniciar interacciones positivas con el sexo opuesto. Ante este aspecto parece que las mujeres tiene más dificultades en las relaciones que se producen en contextos amplios, siendo asertivas e incluso cuando deben interactuar con el sexo opuesto. Estos resultados no son relevantes para la eficacia del curso al no producirse diferencias en la intersección entre titulación y sexo; es decir, independientemente del centro de procedencia la puntuación atendiendo al sexo se mantiene, a la vez que, la mejora tras el mismo es evidente para ambos géneros. 
Torrecilla, Olmos \& Rodríguez: Desarrollo profesional de los docentes de educación secundaria

Tabla 6.

Análisis descriptivo y contraste de hipótesis de los factores de la variable medida

\begin{tabular}{ccccccccc}
\hline & \multicolumn{7}{c}{ Postest } \\
\cline { 2 - 9 } Factores & \multicolumn{2}{c}{ Media } & \multicolumn{7}{c}{ Desviación típica } & \multicolumn{2}{c}{ Media } & \multicolumn{2}{c}{ Desviación típica } \\
\cline { 2 - 9 } & $H$ & $M$ & $H$ & $M$ & $H$ & $M$ & $H$ & $M$ \\
\hline F1 & 70.65 & 56.47 & 22.27 & 23.83 & 81.71 & 53.71 & 14.48 & 30.05 \\
\hline F2 & 57.29 & 54.12 & 30.47 & 31.58 & 61.59 & 54.88 & 28.64 & 28.42 \\
\hline F3 & 60.53 & 41.71 & 27.75 & 31.06 & 68.71 & 63.82 & 24.05 & 26.13 \\
\hline F4 & 60.71 & 57.12 & 31.16 & 32.06 & 78.18 & 50.00 & 18.46 & 30.40 \\
\hline F5 & 29.18 & 30.59 & 25.12 & 27.45 & 56.59 & 50.76 & 26.80 & 29.12 \\
\hline F6 & 63.41 & 60.29 & 27.21 & 29.33 & 76.65 & 54.76 & 27.88 & 28.06 \\
\hline $\begin{array}{c}\text { Percentil } \\
\text { Global }\end{array}$ & 60.88 & 50.41 & 30.51 & 29.33 & 80.53 & 55.82 & 19.57 & 29.02 \\
\hline
\end{tabular}

Fuente: Elaboracion própria

\section{Conclusiones}

La mayor preocupación en la formación permanente debe relacionarse con la eficacia y eficiencia de la actividad formativa seleccionada para el desarrollo de competencias concretas. Cuando uno de los constructos que se pretenden mejorar son las habilidades sociales la relevancia de constatación aumenta. Esta preocupación radica en el hecho de que las habilidades sociales se aprenden de forma longitudinal y se vinculan con los estereotipos sociales atendiendo a roles como el género (Del Prette \& Del Prette, 2001; Ruiz, García \& Rebollo, 2013). Estos aspectos motivan que, en la edad adulta, el nivel de competencia social esté arraigado dificultando el cambio.

A pesar de la complejidad que tiene el trabajo mediante programas para la mejora de las habilidades sociales, son muchos los estudios que han validado la mejora en colectivos adultos ya sean universitarios o profesionales de diferentes campos (Caballo, Guillén \& Salazar, 2008; Fuente, Franco \& Salvador, 2010). El presente estudio supone un avance más en el campo de mejora de las habilidades sociales, al alcanzarse diferencias significativas en docentes de educación secundaria en el nivel global de medida ( $p=.007)$, avalado con un tamaño del efecto entre pequeño y moderado ( $d=$ .457). Si bien es cierto, en esta investigación los principales cambios se apoyan en dos factores: Factor 3 ( $p$.003; $d=.609$ ) y Factor 5 ( $p .000 ; d=.859$ ). Se recuerda que se enfatiza su trabajo en el curso de resolución de conflictos, por lo que se justifican los resultados. Aunque este estudio no es específico de la competencia en habilidades 
sociales, si no que era un componente de la competencia resolución de conflictos, la mejora ha sido evidenciada. Los resultados obtenidos no son solo relevantes para la adquisición de competencias en habilidades sociales y/o en resolución de conflictos, si no que permite corroborar que una combinación de modelos de desarrollo profesional, en este caso curso de formación y colaborativo, permite replicar evidencias de mejora como otros estudios han afirmado mediante diferentes tipos de modelos, trabajando con docentes en temáticas de convivencia (Boqué Torremorell \& García Raga, 2010; Pérez de Guzmán, Amador \& Vargas, 2011).

En lo que respecta al trabajo con docentes, se ha de atender la diversidad del colectivo, lo que conlleva muestras que tengan en cuenta la titularidad de los centros y el género. En esta investigación, la titularidad del centro no ha supuesto un elemento de discrepancia en habilidades sociales; no obstante, en la variable sexo sí se encuentran.

Las diferencias en relación a la variable sexo se fundamentan tanto en el rol género, como en los estereotipos sociales que marcan modelos de crianza diferenciados y, que influyen en las conduzcas de los adultos (Del Prette \& Del Prette, 2001; Ruiz, García \& Rebollo, 2013). Muchos estudios han evidenciado estas diferencias con adultos, llegando a la conclusión de que los hombres son más proclives en habilidades relacionadas con la asertividad, contexto sociales y en relaciones con el sexo opuesto, mientras que las mujeres son más empáticas y emocionales-afectivas (Hermann \& Betz, 2004; Denis, Hamarta \& Ari, 2005). Los factores en los que sobresale el género masculino sobre el femenino se replican en los resultados obtenidos en este estudio mediante la escala de Gismero (2000), sin bien es cierto, en el caso la empatía, emotividad y afectividad, la escala no recoge ítems centrados en las mismas, por lo que no se pueden corroborar dichas afirmaciones. No obstante, la realidad de las diferencias de género se evidencian en los propios baremos del instrumento de medida EHS (2000), en el cual, existe un baremos para hombres y otro para mujeres. Por lo tanto, parece evidente que el nivel de habilidades sociales en los docentes de educación secundaria ha de diferir en función del género.

En esta investigación, tras los resultados obtenidos, la conclusión que se extrae y que corrobora las materializadas en otros estudios es que el diseño de programas específicos para docentes desde el desarrollo profesional supone un recurso para la población que permite un avance significativo en su formación, evidenciándose la eficacia y eficiencia de dicho recurso. 


\section{Referencias Bibliográficas}

Barberá, O. (2010). De nuevo la formación consecutiva y de nuevo el menosprecio a la formación simultánea. En I. González Gallego (Coord.). El nuevo profesor de secundaria. La formación inicial docente en el marco del Espacio Europeo de Educación Superior (pp. 89-96). Barcelona: Graó.

Becker, A. (2000). Basic and Applied Research Methods. Colorado University. Colorado: Spring.

Bolívar, A. (2007). La formación inicial del profesorado de secundaria y su identidad profesional. Estudios sobre Educación, 12, 13-30 [Disponible en http://dspace.si.unav.es/dspace/ bitstream/10171/8987/1/12\%20Estudios\%20Ea.pdf, consultado en 03/08/2013].

Boqué Torremorell, M. C. \& García Raga, L. (2010). Evaluación diferida de la formación del profesorado en convivencia y mediación. Revista Electrónica Interuniversitaria de Formación del Profesorado. REIFOP, 13(3), 87-94. [Disponible en http://www.aufop.com/aufop/uploaded_files/ articulos/1285862366.pdf, consultado en 14/05/2015]

Caballo, V., Guillén, J. \& Salazar, I. (2008). Estilos, rasgos y trastornos de la personalidad: interrelaciones y diferencias asociadas al sexo. PSICO, 40(3), 319-327.

Choi, A. \& Calero, J. (2012). Rendimiento académico y titularidad de centro en España. Profesorado. Revista de currículum y formación del profesorado, 16 (3), 31-57 [Disponible en http://www.redalyc. org/articulo.oa?id=56725002003, consultado en 15/01/2015].

Cohen, J. (1969). Statistical power analysis for the behavioral sciences. New York: Academic Press.

De la Orden, A. \& Jornet, J. (2012). La Utilidad de las evaluaciones de sistemas educativos: el valor de la consideración del contexto. Bordón, 64(2), 69-88.

Del Prette, A. \& Del Prette, Z. (2001). Inventário de Habilidades Sociais: Manual de Aplicação e interpretação e interpretação. São Paulo: Casa do Psicólogo.

Denis, M., Hamarta, E. \& Ari, R. (2005). An investigation of social skills and loneliness levels of university student with respect to their attachment styles in a sample of Turkish students. Social Behavior and Personality, 33(1), 19-32.

Esteve, J.M. (2006). La profesión docente en Europa. Perfil, tendencias y problemática. La formación inicial. Revista de Educación, 340, 19-86 [Disponible en http://www.revistaeducacion.mec.es/ re340/re340_03.pdf, consultado en 15/06/2013).

Eurydice (2003). The Teaching Profession in Europe. Profile, trends and concerns) [Disponible en http:// bookshop.europa.eu/en/key-topics-in-education-in-europe-pbEC3012565/, consultado en 20/05/ 2011].

Fernández Cruz, M. (2006). Desarrollo profesional docente. Granada. Grupo Editorial Universitario.

Fuente, M., Franco, C. \& Salvador, M. (2010). Efectos de un programa de meditación (mindfulness) en la medida de la alexitimia y las habilidades sociales. Psicotema, 22(3), 369-375.

Gismero, E. (2000). EHS. Escala de Habilidades Sociales. Madrid: Tea Ediciones.

González Gallego, I. (2010). Concepto, normativa y propuesta de aplicación para geografía e historia. En I. González Gallego (Coord.). El nuevo profesor de secundaria. La formación inicial docente en el marco del Espacio Europeo de Educación Superior (pp. 27-76). Barcelona: Graó. 
González Sanmamed, M. (2009). Una nueva oportunidad para la formación inicial del profesorado de Educación Secundaria. Revista de Educación, 350, 57-78 [Disponible en http://www. revistaeducacion.educacion.es/re350/re350_03.pdf, consultado en 03/08/2014].

Grossman, P. (1992). Why models matter: An alternative view on professional growth in teaching, Review of Educational Research, 62, 2, 171-179 [Disponible en http://rer.sagepub.com/content/62/2/171. full.pdf, consultado el 15/02/2012].

Hermann, K. \& Betz, N. (2004). Path Models of the Relationships of Instrumentality and Espressiveness to Social Self-Efficacy, Shyness, and Depressive Symptoms. Sex Roles, 51(1/2), 55-66.

Imbernón, F. (2014). Calidad de la enseñanza y formación del profesorado. Un cambio necesario. Barcelona: Octaedro.

Marcelo, C. \& Vaillant, D. (2009). Desarrollo profesional docente. ¿Cómo se aprende a enseñar? Madrid: Narcea.

Martínez Bonafé, J. (2010). Aprender el oficio docente sistematizando la práctica. En A.I. Pérez Gómez (Coord.). Aprender a enseñar en la práctica: procesos de innovación y prácticas de formación en la Educación Secundaria (pp 107-120). Barcelona: Graó.

Pérez de Guzmán, V., Amador, L.V. \& Vargas, M. (2011). Resolución de conflictos en las aulas: un análisis desde la Investigación-Acción. Pedagogía Social. Revista Interuniversitaria 18, 99-114 [Disponible en http://www.upo.es/revistas/index.php/pedagogia_social/article/view/60, consultado el 19/04/2014).

Ruiz, E., García, R. \& Rebollo, M. (2013). Relaciones de género de adolescentes en contextos educativos. Análisis de redes sociales con perspectiva de género. Revista de Currículum y Formación del Profesorado, 17(1), 123-140 [Disponible en http://www.ugr.es/ recfpro/rev171ART8. pdf, consultado el 15/11/2016].

Vidiella Pagés, J. (2011). Aprendiendo a través de la reconstrucción de un curso: formación inicial e identidad docente. En F. Hernández (Coord.). Aprender a ser docente de secundaria (pp. 33-60). Barcelona: Octaedro.

Eva Torrecilla Sánchez

Universidad de Salamanca. Instituto Universitario de Ciencias de la Educación. Facultad de Educación. Departamento de didáctica, organización y métodos de investigación. Email: emt@usal.es

Susana Olmos Migueláñez

Universidad de Salamanca. Instituto Universitario de Ciencias de la Educación. Facultad de Educación. Departamento de didáctica, organización y métodos de investigación. 
Torrecilla, Olmos \& Rodríguez: Desarrollo profesional de los docentes de educación secundaria

M. J. Rodríguez Conde

Universidad de Salamanca. Instituto Universitario de Ciencias de la Educación. Facultad de Educación. Departamento de didáctica, organización y métodos de investigación.

Corresponding Author

Eva Torrecilla Sánchez

Universidad de Salamanca. Instituto Universitario de Ciencias de la Educación. Facultad de Educación. Departamento de didáctica, organización y métodos de investigación. 37008 Salamanca, España

Data de submissão: Janeiro 2017 Data de avaliação: Março 2017 Data de publicação: Dezembro 2017 